\title{
FORTY QUESTIONS ON SINGULARITIES OF ALGEBRAIC VARIETIES*
}

\author{
HERWIG HAUSER ${ }^{\dagger}$ AND JOSEF SCHICHO ${ }^{\ddagger}$
}

Key words. Algebraic Geometry, Analytic Geometry, Singularities, Resolution.

AMS subject classifications. 14B05, 14B12, 32S05, 32S45.

The reader will find in this article a collection of problems, questions and exercises related to the singularities of algebraic and analytic varieties. Many of them are inspired by the work and mathematical conception of Hironaka: they are concrete, involve basic ideas and techniques from geometry and algebra, and they can immediately be attacked from scratch. Some problems rely on or use results proven by Hironaka. Simple and double asterisques indicate the more difficult problems; they may potentially serve as a topic of future research.

\section{Monomials.}

Problem 1. Monomial algebras. The purpose of the exercise is to establish resolution for toric varieties without passing to polyhedral cones, fans and subdivisions, as is done classically. Let $A$ be a set of vectors of non-negative integers in $\mathbb{Z}^{n}$, and let $K$ be a field. For variables $s=\left(s_{1}, \ldots, s_{n}\right)$, write $s^{\alpha}$ for $\prod_{i} s_{i}^{\alpha_{i}}$.

(a) Give a criterion in terms of $A$ for $K[A]=K\left[s^{\alpha}, \alpha \in A\right]$ to be a regular ring.

(b)* Let $\mathcal{A}$ denote the set of all finite and non-empty subsets $A \subset \mathbb{Z}^{n}$. Construct a map $C: \mathcal{A} \rightarrow \mathcal{A}$ with $C_{A} \subset A$ such that the following holds: For any choice of map $\eta: \mathcal{A} \rightarrow \mathbb{Z}^{n}$ with $\eta_{A} \in C_{A}$ and any $A \in \mathcal{A}$, denoting by $A^{\prime}$ the transform

$$
A^{\prime}=A \cup\left\{\alpha-\eta_{A}, \alpha \in C_{A}\right\} .
$$

of $A$ with respect to $C$ and $\eta$, the sequence of transforms $K[A], K\left[A^{\prime}\right], K\left[A^{\prime \prime}\right], \ldots$ becomes eventually regular.

Note. The ring $K[A]$ defines an affine toric, not necessarily normal variety. The map $C$ will prescribe the center of the blowup, the map $\eta$ chooses the chart of the blowup. The transformation rule between $A$ and $A^{\prime}$ is a generalization of the Euclidean division to vectors of integers.

Example. $A=\{(1,1),(1,0),(0,2)\}$ so that $K[A]=K\left[s t, s, t^{2}\right]$ is non-regular. If $C_{A}=A$ and $\eta_{A}=(0,2)$, then $K\left[A^{\prime}\right] \cong K[A]$, whereas for $C_{A}=\{(1,1),(1,0)\}$ and arbitrary $\eta_{A}, K\left[A^{\prime}\right]$ is regular.

References. G. Kempf, F. Knudsen, D. Mumford, B. Saint-Donat, Toroidal Embeddings, Lecture Notes in Math. 339, Springer 1973.

*Received June 1, 2010; accepted for publication June 7, 2011. Supported by project P-21461 of the Austrian Science Fund. It is understood that only a few aspects of Hironaka's œeuvre could be touched on in this article. We recommend the paper "On the Mathematical Work of Professor Heisuke Hironaka", by Lê and Teissier, Publ. RIMS, 44 (2008), pp. 165-177..

†Fakultät für Mathematik, Universität Wien, 1090 Wien, Austria (herwig.hauser@univie.ac.at).

‡Radon Institute, Universität Linz, A-4040 Linz, Austria (josef.schicho@oeaw.ac.at). 
D. Cox, J. Little, H. Schenck, Toric Geometry, forthcoming.

Problem 2*. Monomial Jacobian ideals. This problem aims at a classification of hypersurfaces that admit a purely combinatorial description. For a hypersurface $X$, let $J(X)$ be the ideal generated by the defining function of $X$ and all its partial derivatives. This is the sum of the ideal of $X$ and the Jacobian ideal of the defining equation. Characterize the (germs of) hypersurface singularities $X$ for which $J(X)$ is a monomial ideal.

Note. By the Mather-Yau Theorem and its generalization by Gaffney and Hauser, any germ of a complex analytic hypersurface (of isolated singularity type) is determined up to analytic isomorphism by its singular subspace, defined by the (generally non-radical) ideal $J(X)$. If the ideal $J(X)$ is a monomial ideal, then the singular subspace has a combinatorial description. The simplest examples of singularities with monomial ideals $J(X)$ are Brieskorn singularities $x_{1}^{a_{1}}+\ldots+x_{n}^{a_{n}}=0$ (in this case, the ideal coincides with the Jacibian ideal).

References. J. Mather, S. S.-T. Yau, Classification of isolated hypersurface singularities by their moduli algebras, Invent. Math., 69 (1982), pp. 243-251.

T. Gaffney, H. Hauser, Characterizing singularities of varieties and of mappings, Invent. Math., 79 (1985), pp. 427-447.

H. Hauser, G. Müller, Harmonic and dissonant singularities, in: Proc. Conf. Algebraic Geometry, Berlin 1985 (ed. Kurke et al.), pp. 123-134. Teubner 1986.

Problem 3. Integral closure of monomial ideals. This is a warm-up exercise for getting some acquaintance with the concept of integral closure. For an ideal $I$ of a commutative ring $R$, define the integral closure $\bar{I}$ of $I$ as the elements $x$ of $R$ which satisfy an integrality relation

$$
x^{k}+a_{1} x^{k-1}+\cdots+a_{k-1} x+a_{k}=0
$$

with $a_{i}$ in the $i$-th power $I^{i}$ of $I$.

(a) Show that the integral closure is again an ideal.

(b) Let $R=\mathbb{C}\{x\}$ be the ring of convergent power series in $n$ variables, and let $I$ be generated by $g_{1}, \ldots, g_{m}$. Show that $f \in \bar{I}$ if and only if there is a constant $C>0$ and a neighborhood $U$ of 0 in $\mathbb{C}^{n}$ so that

$$
|f(a)|<C \cdot \max \left\{\left|g_{1}(a)\right|, \ldots,\left|g_{m}(a)\right|\right\}
$$

for all $a \in U$.

(c) Determine the integral closure of an ideal generated by monomials.

Note. An answer to (c) was given by Howald in terms of Newton polyhedra. An alternative, analytic proof of this characterization was found by McNeal and Zeytuncu.

References. J. Howald, Multiplier ideals of monomial ideals, Trans. Amer. Math. Soc., 353 (2001), pp. 2665-2671.

M. Lejeune-Jalabert, B. Teissier, Clôture intégrale des idéaux et équisingularité, Ann. Fac. Sci. Toulouse Math., 17 (2008), pp. 781-859. With an appendix by J.-J. Risler.

J. Lipman, B. Teissier, Pseudo-rational singularities and a theorem of BriançonSkoda about integral closures of ideals, Michigan Math. J., 28 (1981), pp. 97-116.

J. McNeal, Y. Zeytuncu, Multiplier ideals and integral closure of monomial ideals: an analytic approach, preprint 2010, Schrödinger Institute Vienna. 
Problem 4. Ordering monomial ideals. This is an exercise on Hironaka's standard bases. Consider $\mathbb{N}^{n}$ with a total order $\varepsilon$ compatible with addition such that zero is the smallest element. Let $<_{\varepsilon}$ be the induced order on the monomials in $n$ variables.

(a) Show that $<_{\varepsilon}$ induces naturally an order, also denoted by $<_{\varepsilon}$, on the set of monomial ideals in $K\left[x_{1}, \ldots, x_{n}\right]$.

(b) Show that this order is a well-ordering.

(c) For an ideal $I$ of the formal power series $\operatorname{ring} K\left[\left[x_{1}, \ldots, x_{n}\right]\right]$, let $\operatorname{in}_{x}(I)$ be the initial ideal of $I$, given as the ideal generated by all minimal monomials of the expansions of elements of $I$. Show that the minimum $\min (I)$ and the maximum $\max (I)$ of $\operatorname{in}_{x}(I)$ over all coordinates $x_{1}, \ldots, x_{n}$ exist.

(d) Show that $K\left[\left[x_{1}, \ldots, x_{n}\right]\right] / I$ is isomorphic as a $K$-vectorspace to $K\left[\left[x_{1}, \ldots, x_{n}\right]\right] / \operatorname{in}_{x}(I)$.

(e) Let $<_{\varepsilon}$ be compatible with the total degree. Let $g_{1}, \ldots, g_{m}$ be generators of $I$ whose initial monomials generate $\operatorname{in}_{x}(I)$. Show that the strict transform $I^{\prime}$ of $I$ under the blowup of $\mathbb{A}^{n}$ with center 0 at any point $a^{\prime}$ of the exceptional divisor is generated by the strict transforms of $g_{1}, \ldots, g_{m}$.

(f) Show that $\min \left(I^{\prime}\right) \leq_{\varepsilon} \min (I)$.

Note. Generators of ideals as in (e), but taking instead of the initial monomials the initial forms with respect to the natural grading, were called by Hironaka standard basis (and are called nowadays Macaulay basis). Hironaka developed the extension of the Weierstrass Division Theorem to ideals as in (d) in order to construct reduced standard bases, whose orders were then used to define his invariant $\nu^{*}$. At about the same time, Grauert established the Division Theorem for convergent power series, using it for the construction of the semi-universal deformation of an isolated singularity. Proofs for (c) and (f) can be found in Hauser's paper. The inequality of (f) is the analog of Bennett's Theorem asserting the non-increase of the Hilbert-Samuel function under blowup with center along which normal flatness holds.

REFERENCES. B. Bennett, On the characteristic function of a local ring, Ann. Math., 91 (1970), pp. 25-87.

H. Hironaka, Resolution of singularities of an algebraic variety over a field of characteristic zero, Ann. Math., 79 (1964), pp. 109-326.

H. Hauser, Three power series techniques, Proc. London Math. Soc., 88 (2004), pp. 1-24.

B. Singh, Effect of permissible blowing up on the local Hilbert function, Invent. Math., 26 (1974), pp. 201-212.

\section{Polyhedra.}

Problem 5. Lattice points in simplices. Here is a simple counting exercise that has some relation to resolution in positive characteristics. For integers $m, c \geq 1$ consider the simplex $\Delta=\left\{\alpha \in \mathbb{N}^{n},|\alpha|=m\right\}$ and the lattice $L=c \cdot \mathbb{N}^{n}$. Characterize the vectors $r \in \mathbb{Z}^{n} / L$ such that $|(r+\Delta) \cap L|=|(\Delta \cap L)|$. Obviously, the set of these vectors is a union of equivalence classes of the set of vectors of length 0 modulo $L$. How many such vectors do exist?

Note. For $n=3, m=3, c=2$, your answer should be 3 . Counting lattice points in simplices and their translates appears in the theory of wild singularities and kangaroo points. These singularities represent one of the main obstructions to the resolution of singularities in positive characteristic. See also Abhyankar's notion of good points. 
References. S. Abhyankar, Good points of a hypersurface, Adv. Math. ,68 (1988), pp. 87-256.

H. Hauser, Wild singularities and kangaroo points for the resolution of singularities in positive characteristic, preprint 2010.

Problem 6. Newton polyhedra with compact facets. Attempts to define an invariant of hypersurface singularities in terms of their Newton polyhedra has lead us to study this problem.

Let $P \in K[x]$ be a polynomial in $n$ variables and $N$ its Newton polyhedron at a given point $a \in \mathbb{A}^{n}$ (i.e., the positive convex hull in $\mathbb{R}^{n}$ of the exponents of the expansion of $P$ at $a$ ). For a blowup of $\mathbb{A}^{n}$ along a coordinate subspace of codimension $\geq 2$, consider the total transforms $P^{*}$ of $P$ in any of the the affine coordinate charts, together with their associated Newton polyhedra $N^{*}$, taken at the origin of the respective chart.

(a) Determine $N^{*}$ in terms of $N$.

(b) Show that for any sequence of such blowups the transformed polyhedra have eventually no compact facet.

(c)* For a hypersurface $f=0$ in $\mathbb{A}^{n}$, consider the locus of points where the Newton polyhedron of $f$ has in all local coordinates at least one compact facet. Show that this locus is finite.

Note. Newton polyhedra have been studied by Hironaka (and others) in order to control the improvement of singularities under suitable blowups. A hypersurface singularity has normal crossings if and only if its Newton polyhedron is in some local coordinates an orthant. Hypersurface singularities whose Newton polyhedron has a compact facet in all local coordinate systems can be seen as being far away from being normal crossings. It is not clear how to define an invariant which is able to capture this distance from normal crossings in a coordinate independent manner and which improves under blowup until all compact faces have disappeared. Considering only monomial blowups in fixed coordinates, such a measure can be defined and used to prove (b).

References. C. Bruschek, D. Wagner, Some constructions in the étale topology, Expositiones Math., 29:1 (2011), pp. 133-141. 1987.

V. Cossart, Sur le polyèdre caractéristique, Thèse d'État. Univ. Paris-Sud, Orsay

H. Hauser, Three power series techniques, Proc. London Math. Soc., 88 (2004), pp. $1-24$.

H. Hauser, D. Wagner, Alternative invariants for the embedded resolution of surfaces in positive characteristic, preprint 2009.

H. Hironaka, Characteristic polyhedra of singularities, J. Math. Kyoto Univ., 7 (1967), pp. 251-293.

H. Hironaka, Certain numerical characters of singularities, J. Math. Kyoto Univ., 10 (1970), pp. 151-187.

B. Youssin, Newton polyhedra without coordinates, Memoirs Amer. Math. Soc., 433 (1990), pp. 1-74, 75-99.

Problem 7*. Hironaka's polyhedral game. Two player's A and B play a game on a non-empty finite subset $\Gamma$ of $\mathbb{N}^{n}$, where $n \in \mathbb{N}$ is a positive integer ( 3 is a good candidate). Player $A$ specifies a subset $J \subseteq\{1, \ldots, n\}$, followed by player $B$ who selects an element $j \in J$. Then the finite subset $\Gamma$ is replaced by a transformed 
subset, in the following way: the $j$-th coordinate of every point is replaced by the sum of all coordinates with index in $J$. Then player $A$ specifies again a finite subset, and so on. Player A has won if $\Gamma$ has become an orthant, i.e., if it contains a point $\gamma$ such that $\Gamma \subset \gamma+\mathbb{N}^{n}$. Find a winning strategy for A without using induction on the dimension.

Note. The polyhedral game appears in various disguises within resolution of singularities, with various proposals how to win it, the first going probably back to Zariski. When Hironaka formulated his polyhedral game - with a succinct winning strategy proposed soon afterwards by Spivakovsky - it was quickly seen that this game is too coarse to imply resolution of singularities. There then appeared a more exigent version of the game, the hard polyhedral game, which turned out to be too hard - it does not admit a winning strategy. It is believed today that resolution of singularities cannot be reduced to a purely combinatoric problem.

References. M. Spivakovsky, A solution to Hironaka's Polyhedra Game, In: Arithmetic and Geometry. Papers dedicated to I.R. Shafarevich, vol II (eds. M. Artin, J. Tate), Birkhäuser 1983, pp. 419-432.

M. Spivakovsky, A counterexample to Hironaka's 'hard' polyhedra game, Publ. RIMS, Kyoto University, 18 (1983), pp. 1009-1012.

D. Zeillinger, A short solution to Hironaka's polyhedra game, L'Enseign. Mathém, 52 (2006), pp. 143-154.

\section{Transversality.}

Problem 8. Analytic irreducibility. Here are a few problems that should keep $\mathrm{PhD}$ students in algebraic geometry busy for some time. Of course, the students could also gain something: insight, satisfaction over a solved puzzle, and credit for a publication.

(a) Find an effective criterion for checking whether a polynomial in $n$ variables over $\mathbb{C}$ is analytically irreducible at 0 .

(b) Find an effective criterion for checking whether a polynomial in $n$ variables over $\mathbb{C}$ defines locally at 0 a normal crossings divisor, i.e. is analytically equivalent to a monomial.

(c)* Is it true that a free divisor in $\left(\mathbb{C}^{n}, 0\right)$ has normal crossings if and only if the singular subspace (defined by the equation and its partial derivatives) is reduced? Here, a divisor is called free if and only if the module of logarithmic vector fields is free, and a vector field is called logarithmic if and only if the derivative of any multiple of the defining equation is a multiple of the defining equation.

(d)* Which hypersurface singularities have a reduced singular subspace?

(e)* Classify all (not necessarily reduced) curves which may appear as the singular subspace of a surface.

Note. Clearly, (b) is an easy consequence of (a). A divisor is free if the module of analytic vector fields on $\left(\mathbb{C}^{n}, 0\right)$ which are tangent to the divisor is a free module. Such divisors have been introduced and studied by Saito. Tangent vector fields are also called logarithmic, being dual to the logarithmic differential forms.

As for (a), one can consider the normalization of the divisor defined by the polynomial. We have irreducibility if and only if the preimage of zero under the normalization map has exactly one closed point. A more direct approach is via the Artin Approximation Theorem. There exists, for any integer $m$ and any polynomial $f$, a number 
$k$ such that if $f \equiv \bar{g} \cdot \bar{h}$ modulo terms of degree $>k$ for some polynomials $\bar{g}$ and $\bar{h}$ then $f=g \cdot h$ for convergent series $g$ and $h$ coinciding up to degree $m$ with $\bar{g}$ and $\bar{h}$. The problem is to determine the bound $k$ explicitly in terms of $m$ and $f$. This is related to the more general problem of the existence and the computability of the Artin function.

Problem (e) is related to the integral of an ideal studied by Pelikaan. This is the ideal of functions all whose derivatives belong to the given ideal, or, alternatively, the symbolic square of the ideal.

REFEREnCES. M. Artin, On the solution of analytic equations, Invent. Math., 5 (1968), pp. 277-291.

E. Faber, Normal crossings in local analytic geometry, Ph.D. Thesis, Univ. Vienna 2011.

M. Hickel, Un cas de majoration affine pour la fonction d'approximation d'Artin, C. R. Acad. Sci. Paris, 346 (2008), pp. 753-756.

R. Pelikaan, Finite determinacy of funtions with non-isolated singularities, Proc. London Math. Soc., 57 (1988), pp. 357-382.

K. Saito, Theory of logarithmic differential forms and logarithmic vector fields, J. Fac. Sci. Univ. Tokyo, 27 (1980), pp. 265-291.

M. Spivakovsky, Non-existence of the Artin function for Henselian pairs, Math. Ann., 299 (1994), pp. 727-729.

J. Wavrik, A theorem on solutions of analytic equations with applications to deformations of complex structures, Math. Ann., 216 (1975), pp. 127-142.

Problem 9*. Distance to normal crossings. The resolution problem can be formulated as the problem of constructing a sequence of blowups such that the total transform becomes normal crossing. This motivates the following question.

Find a (significant) measure for the distance of a hypersurface singularity from having normal crossings. This measure should not increase under point blowup.

Note. The condition on point blowups comes from the observation that Hironaka's invariants generally do not increase if the center is chosen too small (but one may have problems to achieve an actual decrease). Another argument is that the total transforms of normal crossings divisors under point blowup are again normal crossings.

This problem is related to problem (6). Any reasonable resolution invariant should not increase under point blowup (though, in general, it will only decrease if the center of the blowup is sufficiently large). Most proposals in the literature try to capture the monomiality of a function by factoring it into a monomial part (usually given as the defining equation of the exceptional divisor produced by earlier blowups) and a singular non monomial part, whose multiplicity is then taken as the required measure. It is known by examples of Moh that in positive characteristic this invariant does not behave well under descent in dimension (i.e., when passing to coefficient ideals) and taking the transform of the coefficient ideal under blowup.

Already for plane curves the problem is interesting and consists in finding a substitute for the usual invariant given as the lexicographic pair formed by the local multiplicity and the maximal slope of the first segment of the Newton polygon.

RefEREnCES. T.-T. Moh, On a stability theorem for local uniformization in characteristic p, Publ. Res. Inst. Math. Sci., 2 (1987), pp. 965-973. 
T.-T. Moh, On a Newton polygon approach to the uniformization of singularities of characteristic p, in: Algebraic Geometry and Singularities. Proc. Conference on Singularities, La Rábida. Birkhäuser 1996.

H. Hauser, On the problem of resolution of singularities in positive characteristic (Or: A proof that we are still waiting for), Bull. Amer. Math. Soc., 47 (2009), pp. $1-30$.

Problem 10. Mikado singularities. A union of smooth varieties is called mikado if all intersections of its components are scheme-theoretically smooth. Mikado is a natural generalization of normal crossings. It is inspired by the geometry of hyperplane arrangements.

(a) Construct an example of two analytically non-isomorphic mikado hypersurface singularities of the same dimension and having the same number of components.

(b) Construct a union of smooth hypersurfaces whose pairwise scheme-theoretic intersections are smooth, but which is not mikado.

(c) Find a mikado hypersurface singularity which is not analytically isomorphic to a hyperplane arrangement.

(d)* Find a characterization of mikado singularities through unions of linear spaces.

Note. Clearly, any union of linear spaces is mikado, as well as any union of smooth plane curves meeting pairwise transversally. An answer to (a) was given by Whitney, taking unions of four lines in the plane passing through a given point, with different cross ratios.

References. D. De Concini, C. Procesi, Wonderful models of subspace arrangements, Selecta Math., 3 (1995), pp. 459-494.

L. Li, Wonderful compactifications of arrangements of subvarieties, Michigan Math. J., 58 (2009), pp. 535-563.

E. Faber, H. Hauser, Today's Menu: Geometry and resolution of singular algebraic surfaces, Bull. Amer. Math. Soc., 47 (2010), pp. 373-417.

Problem 11. Blowup of mikado. This exercise shows that it is not a straightforward matter to replace normal crossings by mikado schemes in the resolution process. Show that the blowup of a mikado scheme in $\mathbb{A}^{n}$ along a center $Z \subset \mathbb{A}^{n}$ transversal to all its components need not be again mikado.

Note. Make first precise what could be meant by transversal (one possible option is that the union of the variety and the center is again mikado). The instability of mikado under blowup was observed by $\mathrm{Li} \mathrm{Li}$.

REFERENCES. L. Li, Wonderful compactifications of arrangements of subvarieties, Michigan Math. J., 58 (2009), pp. 535-563.

E. Faber, H. Hauser, Today's Menu: Geometry and resolution of singular algebraic surfaces, Bull. Amer. Math. Soc., 47 (2010), pp. 373-417.

Problem 12*. Resolution of mikado. Find for any mikado scheme an (explicitly given) birational proper map which transforms the scheme into normal crossings.

Note. Compare this with the resolution of hyperplane arrangements, respectively wonderful models as proposed by Li Li. From a geometric viewpoint, mikado singularities are suitable final forms for the resolution of singular varieties, but algebraically, normal crossings are much easier to handle. The problem exhibits this difference. 
References. G. Goward, A simple algorithm for the principalization of monomial ideals, Trans. Amer. Math. Soc., 357 (2005), pp. 4805-4812.

L. Li, Wonderful compactifications of arrangements of subvarieties, Michigan Math. J., 58 (2009), pp.535-563.

Problem 13. Non normal crossings locus. This exercise exhibits a sublety in the definition of normal crossings. In (8)(b), we gave the definition of analytic normal crossings. A divisor is called algebraically normal crossings if for each of its points, there is a regular system of parameters such that the divisor can be defined locally by a product of (some of) the parameters.

(a) Find an example of a singularity where the algebraic and the analytic non normal crossings locus differ.

(b)* Show that any variety admits an embedded resolution with centers inside the algebraic non normal crossings locus.

(c) Show that this is not the case for the analytic non normal crossings locus.

Note. As an embedded resolution aims at transforming all singularities into normal crossings it is natural to expect that this can be achieved by blowups along centers which lie inside the non normal crossings locus. However, as mentioned by Kollár, there is a slight difference between the algebraic and analytic setting.

ReFEREnCES. J. Kollár, Semi log resolutions, arXiv:0812.3592v1.

Problem 14**. Casas-Alvero conjecture. We find it intriguing because it is a simple algebraic questions on polynomials, but yet still open. Let $P$ be a univariate polynomial over a field of characteristic zero. Assume that each (non constant) derivative shares a divisor with $P$.

(a) Is $P$ a monomial $(a x+b)^{k}$ ?

(b) What could be the respective statement for multivariate polynomials?

Note. The conjecture was proposed by Casas-Alvero. The common divisors may a priori be different for each derivative. A proof for polynomials of prime degree (and several more cases) was given by Graf von Bothmer, Labs, Schicho and van de Woestijne. For positive characteristic, there exist easy counterexamples. For fixed degree, it is easy to set up a system of equations which has a solution over $K$ if and only if there is a counter-example.

References. E. Casas-Alvero, Singularities of plane curves, London Math. Soc. Lect. Notes 276, Cambridge Univ. Press 2000.

H. C. Graf von Bothmer, O. Labs, J. Schicho, C. van de Woestijne, The CasasAlvero conjecture for infinitely many degrees, J. Algebra, 316 (2007), pp. 224-230.

\section{Singular locus.}

Problem 15. Singular singular locus. For illustrative purposes, we wanted to find an easy example of a surface with a singular curve in its singular locus. This lead us to the following question. (a) Construct a complex algebraic surface $X$ whose singular locus $S=\operatorname{Sing}(X)$ (i.e., the support of the singular subspace) equals the curve parametrized by $\left(t^{3}, t^{4}, t^{5}\right)$.

(b)* Answer (a) by a surface whose generic transversal plane section along $S$ is an ordinary cusp $x^{2}=y^{3}$.

(c) Is it possible to realize (a) by a surface whose singular subspace is reduced? 
Note. Any reduced hypersurface with an equation in the square of the ideal of $C$ has $C$ in its singular locus, but the singular locus might be bigger. Following an idea of R. Bryant, you may try for (a) with a quasi-homogeneous equation $f$, using that $f$ belongs to $m \cdot j(f)$, where $m$ denotes the maximal ideal generated by the coordinates and $j(f)$ the Jacobian ideal.

Reference. E. Faber, H. Hauser, Today's Menu: Geometry and resolution of singular algebraic surfaces, Bull. Amer. Math. Soc., 47 (2010), pp. 373-417.

Problem 16. Symmetric singularities. The following exercise should lead to an understanding of the difficulties of resolution imposed by symmetries.

(a) Construct an algebraic surface $X$ in $\mathbb{A}^{3}$ whose singular locus is a union of two or three coordinate axes, say e.g. $\operatorname{Sing}(X)=V(x y, z)$, and so that $X$ is invariant under the linear map exchanging $x$ with $y$.

(b) Can you achieve that the local multiplicity is constant equal to 2 along $\operatorname{Sing}(X)$ ?

(c)* Find a non reduced ideal structure on a given union of coordinate subspaces of $\mathbb{A}^{n}$ so that the blowup of $\mathbb{A}^{n}$ with center this ideal gives a smooth transform.

Note. The type of singularities as in (a) causes problems when trying to resolve them, because, due to the symmetry, one does not know which component of the singular locus to prefer as the first center of blowup. This is usually handled by instead blowing up the origin in order to separate the two local branches of the singular locus. Then, after this preparation, each component of the singular locus can be taken simultaneously as the center of the next blowup. Observe here that if the type of the singularity would have reappeared after the first point blowup, one component of the singular locus would lie in the exceptional divisor, which allows to break the symmetry.

Allowing centers with mild singularities (e.g., normal crossings) could be convenient for resolution purposes. Rosenberg and Faber-Westra have described suitable non reduced ideals defining normal crossings and giving smooth blowups.

In characteristic 0 , there is an obstacle for (b) in the case that the singular locus consists of all three coordinate axes. See also problem 23.

REFERENCES. H. Hauser, Excellent surfaces and their taut resolution, In: Resolution of Singularities, Progress in Math. 181, Birkhäuser 2000.

J. Rosenberg, Blowing up non reduced subschemes of $\mathbb{A}^{n}$, unpublished manuscript 1998.

E. Faber, D. Westra, Blowups in tame monomial ideals, J. Pure Appl. Algebra, 215 (2011), pp. 1805-1821

Problem 17. Smooth singular locus. This is a geometric version of exercise (2) (but easier).

Characterize the hypersurface singularities $f=0$ whose singular locus is schemetheoretically smooth.

Note. Use the Theorem of Mather-Yau and Gaffney-Hauser.

References. J. Mather, S. S.-T. Yau, Classification of isolated hypersurface singularities by their moduli algebras, Invent. Math., 69 (1982), pp. 243-251.

T. Gaffney, H. Hauser, Characterizing singularities of varieties and of mappings, Invent. Math., 79 (1985), pp. 427-447. 


\section{Resolution.}

Problem 18. Affine real resolution. Our efforts to visualize resolutions lead us to the following problem.

(a)* Classify the real algebraic surfaces $X$ in $\mathbb{A}_{\mathbb{R}}^{3}$ which admit an affine resolution, i.e., a smooth algebraic surface $X^{\prime}$ in $\mathbb{A}_{\mathbb{R}}^{3}$ and a birational morphism $\pi: X^{\prime} \rightarrow X$ which is "real proper", i.e., the induced map on subsets of $\mathbb{R}^{3}$ is proper in the Euclidean topology.
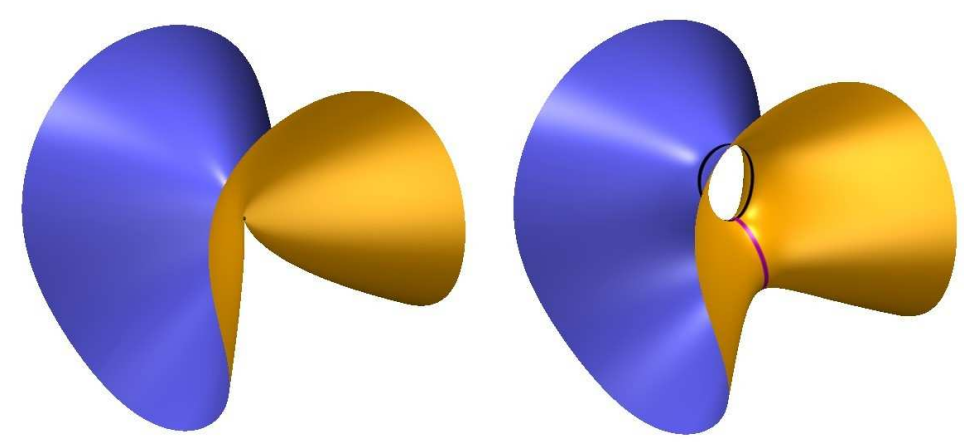

FiguRE 1. An $\mathrm{A}_{2}$-singularity and its real resolution. The exceptional locus is the union of two-2-curves intersecting transversally.

(b)* Is it also possible to define a deformation of $X$ such that the general fiber is isomorphic (as an embedded real analytic surface) to $\widetilde{X}$ (smoothing)?

Note. A typical example is the cone $x^{2}+y^{2}=z^{2}$ whose resolution is the cylinder $x^{2}+y^{2}=1$ induced by the affine map $\mathbb{A}_{\mathbb{R}}^{3} \rightarrow \mathbb{A}_{\mathbb{R}}^{3}$ sending $(x, y, z)$ to $(x z, y z, z)$. The respective smoothing is given by the family $x^{2}+y^{2}=t+z^{2}$. A more interesting example is the $A_{n}$-singularity $x^{2}-y^{2}=z^{n+1}$ with the "real smoothing" $x^{2}-y^{2}=$ $z(z-t)(z-2 t) \cdots(z-n t)$. The exceptional divisors are the compact components of the intersections with the planes $x=0$ and $y=0$.

A necessary criterion for a resolution to be affine real in the above sense is that the self intersection numbers of the real exceptional divisors are even. This follows from the fact that smooth hypersurfaces in $\mathbb{R}^{3}$ are orientable, so they cannot contain closed curves with an odd self intersection number. This also shows that any affine real resolution is real isomorphic to the minimal resolution.

Reference. P. Hacking, Y. Prokhorov, Smoothable Del Pezzo surfaces with quotient singularities, Compos. Math., 146 (2010), pp. 169-192.

Problem 19. Drop of order under blowup. An amusing and easy observation. Let $X$ be a plane curve singularity and consider a sequence of monomial point blowups (i.e., the successive centers are the origins of the affine charts of the preceding blowup).

(a) Show that the local multiplicity of $X$ drops at least to its half if there appears in the sequence of blowups at least one chart change.

(b) Take now a surface in three-space and three monomial point blowups in three different charts (i.e., the final point is the intersection point of the three exceptional components). How does the local multiplicity behave? 
Reference. H. Hauser, D. Wagner, Alternative invariants for the embedded resolution of surfaces in positive characteristic, preprint 2009.

Problem 20. Separation of varieties. A special type of singularity is the intersection locus of two varieties. The following exercise is aiming at resolving this type of singularities.

Let $X$ and $Y$ be algebraic, possibly singular affine varieties in $\mathbb{A}_{\mathbb{C}}^{n}$. Find a sequence of blowups in smooth centers contained in $X \cap Y$ (respectively the transforms of the intersection) which separates $X$ and $Y$.

Note. If $X$ and $Y$ are smooth, just apply principalization to the ideal $I_{X}+I_{Y}$ defining the intersection $X \cap Y$. In general, take a suitably weighted sum $I_{X}^{a}+I_{Y}^{b}$ of $I_{X}$ and $I_{Y}$ so that both powers of the ideals have the same maximal local multiplicity (suggestion of H. Hironaka and O. Villamayor).

References. S. Encinas, H. Hauser, Strong resolution of singularities in characteristic zero, Comment. Math. Helv., 77 (2002), pp. 421-445.

H. Hironaka, Idealistic exponents of singularity, In: Algebraic Geometry, The Johns Hopkins Centennial Lectures, Johns Hopkins University Press 1977.

O. Villamayor, Patching local uniformizations, Ann. Scient. Éc. Norm. Sup. Paris, 25 (1992), pp. 629-677.

Problem 21**. Higher Nash modifications. A single Nash modification does in general not achieve resolution, for instance because branches with the same tangent space are not separated. This motivates the following question.

Nash modifications of hypersurfaces are defined as the blowups at the Jacobian ideal. Geometrically, they are obtained by replacing the singular locus of a hypersurface by the projectivized first jet space. Extend this to higher jet spaces.

Note. The first jet space is just the tangent bundle. For higher jet spaces, it is a priori not clear how to projectivize suitably, i.e., how to take limits of jets.

References. G. Gonzalez-Sprinberg, Désingularisation des surfaces par des modifications de Nash normalisées, Sém. Bourbaki 1985/86. Astérisque, 145-146 (1987), pp. 187-207.

H. Hironaka, On Nash blowing-up, In: Arithmetic and Geometry II. Progr. Math. 36. Birkhäuser 1983, pp. 103-111.

A. Nobile, Some properties of the Nash blowing-up, Pacific J. Math., 60 (1975), pp. 297-305.

M. Spivakovsky, Sandwiched singularities and desingularization of surfaces by normalized Nash transformations, Ann. Math., 131 (1990), pp. 411-491.

T. Yasuda, Higher Nash blowups, Compos. Math., 143 (2007), pp. 1493-1510.

J. A. Moody, On resolving singularities, J. London Math. Soc., 64 (2001), pp. $548-564$.

Problem 22*. Global descent for resolution. Hiranaka-type resolution uses induction on the dimension: one constructs singularity objects in lower codimension. The existing constructions are local, and this leads to the necessity of proving that the local constructions induce globally defined centers.

Construct a globally defined descent object for the resolution of singularities in characteristic zero. 
Note. The classical descent via hypersurfaces of maximal contact is necessarily local, by the only local existence of these hypersurfaces. Włodarczyk showed how to make the local descents intrinsic up to analytic isomorphsims by using homogenized coefficient ideals. Using modules of derivations and differential operators there is a certain chance to construct globally defined objects which give a substitute for the local descent.

REFERENCE. H. Hironaka, Theory of infinitely near singular points, J. Korean Math. Soc., 40 (2003), pp. 901-920.

Problem 23*. Failure of maximal contact. An obvious necessary condition for a hypersurface of maximal contact is that the locus of maximal multiplicity lies in a hypersurface. This condition fails in positive characteristics.

Characterize all hypersurfaces in $\mathbb{A}_{K}^{n}, K$ a field of positive characteristic, whose locus of points of maximal multiplicity has local embedding dimension $n$ at 0 .

Note. The first example was given by Narasimhan, of equation $x^{2}+y^{3} z+$ $z w^{3}+w y^{7}=0$ over a field of characteristic 2 . The equimultiple locus is the curve parametrized by $\left(t^{32}, t^{7}, t^{19}, t^{15}\right)$. Note that this example also shows that there need not always exist locally at a singular point a smooth hypersurface whose transforms contain all points where the local multiplicity has remained constant. Namely, any such hypersurface through 0 would get separated from the above curve under a sequence of point blowups, while the multiplicity must remain the same for semicontinuity reasons (it remains constant along the curve).

REFERENCES. R. Narasimhan, Monomial equimultiple curves in positve characteristic, Proc. Amer. Math. Soc., 89 (1983), pp. 402-413.

R. Narasimhan, Hyperplanarity of the equimultiple locus, Proc. Amer. Math. Soc., 87 (1983), pp. 403-406.

H. Hauser, Seventeen obstacles for resolution of singularities, In: The Brieskorn Anniversary Volume, Progress in Math. 162, Birkhäuser 1997.

S. Mulay, Equimultiplicity and hyperplanarity, Proc. Amer. Math. Soc., 87 (1983), pp. 407-413.

Problem 24**. Quings. In characteristic $p$, it is natural to consider polynomials modulo $p$-th powers, because $p$-th powers of a descent object can be removed by an automorphism of the original resolution object.

(a) Let $K$ be perfect. Show that the surface $x^{p}+y^{p} z=0$ is locally at each point of the $z$-axis isomorphic to its germ at 0 .

(b) Let $K$ be a field of characteristic $p>0$. Develop a reasonable concept of local multiplicity for hypersurfaces in an equivalence class of power series in $K[[x]] / K[[x]]]^{p}$.

(c) Resolve "plane curves" $f$ in $K[[y, z]] / K[[y, z]] p$.

Note. Taking in (b) the maximum order of a representative in $K[[x]]$ of elements in $K[[x]] / K[[x]]^{p}$ yields an invariant which is not upper semicontinuous (as observed, among others, by Hironaka).

References. H. Hironaka, Program for resolution of singularities in characteristics $p>0$, Notes from lectures at the Clay Mathematics Institute, September 2008.

H. Hauser, D. Wagner, Alternative invariants for the embedded resolution of surfaces in positive characteristic, preprint 2009. 
Problem 25**. Monomialization of morphisms. This is a relative formulation of the resolution problem. Show that any morphism of varieties over a field of characteristic zero can be transformed via blowups of source and target into a monomial morphism, i.e., a morphism which can be expressed in suitable local coordinates by monomials.

Note. There have been important recent advances by Cutkosky and Abramovich, Karu, Matsuki and Włodarcyzk. In positive characteristic there are simple counterexamples.

References. D. Abramovich, K. Karu, M. Matsuki, J. Włodarczyk, Torification and factorization of birational maps, J. Amer. Math. Soc., 15 (2002), pp. 531-572.

D. Cutkosky, Local monomialization and factorization of morphisms, Astérisque, 260 (1999).

D. Cutkosky, Toroidalization of Dominant Morphisms of 3-Folds, Memoirs Amer. Math. Soc., 890 (2007).

D. Cutkosky, Monomialization of Morphisms from 3-folds to surfaces, Lecture Notes in Math. 1786, Springer 2002.

\section{Algebraic series.}

Problem 26. Algebraic series. The purpose of the following exercise is to providesome familiarity with multivariate algebraic power series. A formal power series is called algebraic or Nash if it is an algebraic element over the polynomial ring, i.e., satisfies a polynomial equation in one variable with polynomial coefficients. The simplest examples are $(1+x)^{-1}$ and $\sqrt{1+x}$.

(a) Show that any complex algebraic power series in $n$ variables is convergent in some neighborhood of zero in $\mathbb{C}^{n}$.

(b)* Show that for any algebraic power series $h$ with $h(0)=0$ there is a polynomial mapping $F: \mathbb{C}^{n+p} \rightarrow \mathbb{C}^{p}, F(0)=0$, with non zero Jacobian determinant $\operatorname{det}\left(D_{y} F(0)\right) \neq 0$ (where $y=\left(y_{1}, \ldots, y_{p}\right)$ are the coordinates on $\left.\mathbb{C}^{p}\right)$ such that the first component $y_{1}$ of the implicit solution $y(x)$ of $F(x, y)=0$ equals $h$.

Note. For (a), you may want to find a suitable dominating series. For (b), normalize the variety defined by the minimal polynomial of $h$ and use Zariski's Main Theorem.

References. J. Bochnak, M. Coste, M.-F. Roy, Real Algebraic Geometry, Springer 1998.

J. P. Lafon, Séries formelles algébriques, C. R. Acad. Sci. Paris, 260 (1965), pp. 3238-3241.

M. Raynaud, Anneaux locaux henséliens, Lecture Notes in Math. 169. Springer 1970.

J. Ruiz, The basic theory of power series, Vieweg 1993.

Problem 27. Recursions for algebraic series. If the sequence of coefficients satisfy a recursion formula, then this formula gives another way of representing the power series in finite terms.

(a) Let $S=\sum_{k} a_{k} x^{k}$ be a univariate algebraic power series over $\mathbb{C}$. Prove that there exists a recursion formula for the coefficients $a_{k}$.

(b) Same problem for $D$-finite power series. A power series is called D-finite if and only if it satisfies an ordinary differential equation with polynomial coefficients. 
References. R. Stanley, Differentiably finite power series, European J. Combin., 1 (1980), pp. 175-188.

J. Denef, L. Lipshitz, Power series solutions of algebraic differential equations, Math. Ann., 267 (1984), pp. 213-238.

J. Denef, L. Lipshitz, Algebraic power series and diagonals, J. Number Theory, 26 (1987), pp. 46-67.

Problem 28. Artin Approximation. Here is a(nother) classical topic.

(a) Let $X$ and $Y$ be isolated hypersurface singularities in $\left(\mathbb{C}^{n}, 0\right)$. Show that if $X$ and $Y$ are isomorphic up to a sufficiently high power of the maximal ideal, then $X$ and $Y$ are analytically isomorphic.

(b)** Find a proof for the Artin Approximation Theorem which does not use induction on the dimension. Such a proof should make explicit why the counterexamples of Gabrielov (separate variables condition for solutions of polynomial equations) and Becker (nested subring condition for solutions of non-algebraic series) cannot be avoided.

Note. Assertion (a) was proven by Hironaka and Rossi before Artin had proved his approximation theorem (which, obviously, gives (a)). Artin's proof relies on a multiple use of the Weierstrass Division Theorem. This involves in each application an analytic coordinate change which need not maintain the nested subring or the separatedness condition. Popescu's and Spivakovsky's proofs for systems of algebraic equations whose solutions satisfy the nested subring condition are very involved.

References. M. André, Artin's theorem on the solution of analytic equations in positive characteristic, Manuscripta Math., 15 (1975), pp. 314-348.

M. Artin, On the solution of analytic equations, Invent. Math., 5 (1968), pp. $277-$ 291.

M. Artin, Algebraic approximation of structures over complete local rings, Publ. Math. Inst. Hautes Études Sci., 36 (1969), pp. 23-58.

J. Becker, A counterexample to Artin approximation with respect to subrings, Math. Ann., 230 (1977), pp. 195-196.

A. Gabrielov, The formal relations between analytic functions, Funkts. Anal. Prilozh., 5 (1971), pp. 64-65.

M. Greenberg, Rational points in Henselian discrete valuation ring, Publ. Math. Inst. Hautes Études Sci., 31 (1966), pp. 59-64.

H. Hironaka, H. Rossi, On the equivalence of imbeddings of exceptional complex spaces, Math. Ann., 156 (1964), pp. 313-333.

T. Ogoma, General Néron desingularization based on the idea of Popescu, J. Algebra, 167 (1994), pp. 57-84.

D. Popescu, General Néron desingularization, Nagoya Math. J., 100 (1985), pp.97-126.

D. Popescu, General Néron desingularization and approximation, Nagoya Math. J., 104 (1986), pp. 85-115.

D. Popescu, Polynomial rings and their projective modules, Nagoya Math. J., 113 (1989), pp. 121-128.

M. Spivakovsky, A new proof of D. Popescu's theorem on smoothing of ring homomorphisms, J. Amer. Math. Soc., 294 (1999), pp. 381-444.

B. Teissier, Résultats récents sur l'approximation des morphisms en algèbre commutative (d'après Artin, Popescu, André, Spivakovsky), Sém. Bourbaki, 784 (1994), pp. $1-15$. 
J. Wavrik, A theorem on solutions of analytic equations with applications to deformations of complex structures, Math. Ann., 216 (1975), pp. 127-142.

Problem 29. Division of algebraic series. This exercise demonstrates that the set of algebraic power series is not closed under division (with remainder).

(a) Divide $x y$ by $\left(x-y^{2}\right)\left(y-x^{2}\right)$ as a power series in $K[[x, y]]$, taking for the divisor the initial monomial $x y$. More precisely, write $\left(x-y^{2}\right)\left(y-x^{2}\right)=x y Q+R$, with $Q, R \in K[[x, y]]$ and no monomial summand of $R$ is divisible by $x y$. What do you observe?

(b)* Let $f \in K\left[\left[x_{1}, \ldots, x_{n}\right]\right]$ be an $x_{n}$-regular algebraic series (i.e., $f\left(0, \ldots, 0, x_{n}\right)$ $\neq 0)$. Show that the Weierstrass division of any algebraic series by $f$ yields an algebraic quotient and remainder. (The Weierstrass division is defined by requiring that no monomial summand of the remainder is divisible by the leading monomial of $f\left(0, \ldots, 0, x_{n}.\right)$

(c) Find suitable functional equations for the series $\sum_{k>0} x^{2^{k}}$ and $\sum_{k>0} x^{k^{2}}$.

(d) Divide $x y$ by the series $x y-z(1+y)\left(1+x^{2} y\right)$ with initial monomial $x y$ as in (a). Is the remainder an algebraic series?

Note. According to Hironaka, the example from (a) is due Gabber-Kashiwara. It yields a lacunary series as the remainder of the division which therefore is not algebraic. This and similar examples were studied in combinatorics by BousquetMélou and Petkovšek as generating functions for counting walks in lattices. Lafon proved (b), and Hironaka extended the division to ideals of algebraic series in the sense of the Grauert-Hironaka-Galligo Division Theorem, assuming that the initial ideal satisfies a natural generalization of $x_{n}$-regularity.

References. J. P. Lafon, Séries formelles algébriques, C. R. Acad. Sci. Paris, 260 (1965), pp. 3238-3241.

H. Hironaka, Idealistic exponents of singularity, In: Algebraic Geometry, The Johns Hopkins Centennial Lectures. Johns Hopkins University Press 1977.

M. Bousquet-Mélou, M. Petkovšek, Linear recurrences with constant coefficients: the multivariate case, Discrete Mathematics, 225 (2000), pp. 51-75.

Problem 30**. Algebraic solutions of ODE's (Grothendieck's p-Curvature Conjecture). Let $a_{i}(x) \in \mathbb{Q}[x]$ be polynomials in one variable $x$, and consider the ordinary differential equation

$$
D: a_{k} y^{(k)}+a_{k-1} y^{(k-1)}+\cdots+a_{1} y^{\prime}+a_{0} y=0 .
$$

Then $D$ has a complete set of algebraic series solutions if and only if, for almost all primes $p$, the reduction $D_{p}$ of $D$ modulo $p$ has a complete set of rational solutions in $\mathbb{F}_{p}(x)$.

Note. The conjecture is still wide open. The name is due to the formulation of the conjecture in terms of the associated Galois group. In the discrete case, i.e., difference equations, there is a formulation and proof of the conjecture by Di Vizio.

References. N. Katz, A conjecture in the arithmetic theory of differential equations, Bull.Soc. Math. France, 110 (1982), pp. 203-239 and pp. 347-348.

L. Di Vizio, On the arithmetic theory of q-difference equations. The q-analogue of the Grothendieck-Katz's conjecture on p-curvatures, Invent. Math., 150 (2002), pp. $517-578$. 


\section{Symmetry.}

Problem 31**. Symmetries of hypersurfaces. The following is one of the main challenges in Cauchy-Riemann geometry, and even for manifolds no complete answer is known.

(a) Let $X$ be the germ of a smooth real analytic hypersurface in $\left(\mathbb{C}^{n}, 0\right)$. Describe the group of local biholomorphic automorphisms of $\left(\mathbb{C}^{n}, 0\right)$ fixing $X$.

(b) Determine the relation of the holomorphic automorphism group of $X$ with its Lie algebra.

(c) Same problem as in (a), with $X$ singular.

Note. The subgroup of $\operatorname{Aut}\left(\mathbb{C}^{n}, 0\right)$ of ambient analytic automorphisms fixing $X$ is an infinite dimensional Lie group whose Lie algebra consists of the germs of analytic vector fields on $\left(\mathbb{C}^{n}, 0\right)$ which are tangent to $X$. It was shown by Hauser and Müller that for $n \geq 3$ the group as well as its Lie algebra determine $X$ up to isomorphism.

REFERENCES. S. Chern, J. Moser, Real hypersurfaces in complex manifolds, Acta Math., 133 (1974), pp. 219-271.

H. Hauser, G. Müller, Affine varieties and Lie algebras of vector fields, Manuscr. Math., 80 (1993), pp. 309-337.

H. Hauser, G. Müller, A rank theorem for analytic maps between power series spaces, Publ. Math. IHES, 80 (1994), pp. 95-115.

B. Lamel, N. Mir, Parametrization of local CR automorphims by finite jets and applications, J. Amer. Math. Soc., 20 (2007), pp. 519-572.

Problem 32. Symmetries of product singularities. The question below has its origin in mere curiousity: is the symmetry of a product singularity determined by the symmetry of the factors?

(a) Determine the local symmetries of the Cartesian square $C \times C$ of the cusp $C: x^{2}=y^{3}$ at 0 . A local symmetry of a variety $X \subset \mathbb{C}^{n}$ is an analytic isomorphisms of the germ of $\mathbb{C}^{n}$ at 0 fixing $X$.

(b)* Is it possible to construct the automorphism group of a Cartesian product from the automorphisms of the factors?

Reference. H. Hauser, G. Müller, Automorphisms of direct products of algebroid spaces, In: Singularity Theory and its Applications, Warwick 1989, Part I Springer Lecture Notes in Math. 1462, 1991.

Problem 33. Enzensberger's Star. The Astroid $x^{2 / 3}+y^{2 / 3}=1$ is the real plane curve (hypocycloid) parametrized by $(\cos (3 t), \sin (3 t))$. It equals the trajectory of a point on a small circle rolling inside a larger circle.

(a) Determine the ratio of radii and the polynomial equation of the Astroid.

(b) Find an analoguous compact real algebraic surface in $\mathbb{R}^{3}$.

(c) Answer (b) by requiring the surface to have isolated singularities.

(d)* Find a construction recipe for this surface analogous to the rolling circles.

Note. The equation $x^{2 / 3}+y^{2 / 3}=1$ with rational exponents can be replaced by a polynomial equation by raising it twice to the third power. In three variables, it is a bit more work to find the polynomial equation for $x^{2 / 3}+y^{2 / 3}+z^{2 / 3}=1$. Note that this solves (b) but not (c).

The solution of (c) has lead to the design of a surface with the purpose of honouring the German author and poet Hans-Magnus Enzensberger. 
Reference. A. Fritz, H. Hauser, Platonic Stars, Math. Intelligencer 32, 2 (2010), pp. 23-36.

\section{Miscellanea.}

Problem 34. Polynomially defined singularities. We think that the second question is natural, but we do not know the answer.

(a) Show that any complex analytic hypersurface singularity can be defined by a polynomial in as many variables as the codimension of the singular locus indicates, with coefficients convergent power series in the remaining variables.

(b)* Is any complex analytic hypersurface singularity algebraic, i.e., analytically isomorphic to a singularity that can be defined by a polynomial?

Note. (a) is easy for isolated singularities. The general case of (a) is due to Shiota. Question: Is it possible to define an even more precise normal form, e.g., $f$ having tail $(=f$ minus initial monomial) in the direct complement of its jacobian ideal? Compare this with Arnold's classification of simple singularities.

References. V. Arnold, Singularity Theory, Cambridge University Press.

T. De Jong, G. Pfister, Local Analytic Geometry, Advanced Lectures Math., Vieweg 2000.

M. Shiota, Equivalence of differentiable mappings and analytic mappings, Publ. Math. Inst. Hautes Études Sci., 54 (1981), pp. 37-122.

Problem 35**. Plain varieties. Another natural question which we do not know how to answer.

Call a complex algebraic variety plain if it is everywhere locally (in the Zariski topology) isomorphic to an open subset of affine space. Is plain equivalent to smooth and rational?

Note. Clearly, every plain variety is smooth and rational. The converse is true for curves and surfaces.

References. G. Bodnár, H. Hauser, J. Schicho, O. Villamayor, Plain varieties, Bull. London Math. Soc., 40 (2008), pp. 965-971.

Problem 36**. Strong factorization (an open problem). Show that any projective birational morphism between non singular varieties is a sequence of blowups along non singular subvarieties followed by a sequence of blowdowns to non singular subvarieties.

Note. The factorization by an arbitrary sequence of blowups and blowdowns, known as the Weak Factorization Theorem, was proven by Włodarczyk, extending ideas of Morelli. For surfaces, see Hartshorne V.5.4.

References. D. Abramovich, K. Karu, K. Matsuki, J. Włodarczyk, Torification and factorization of birational maps, J. Amer. Math. Soc., 15 (2002), pp. 531-572.

D. Cutkosky, Local factorization of birational maps, Adv. Math., 132 (1997), pp. $167-315$.

D. Cutkosky, Local monomialization and factorization of morphisms, Astérisque, 260 (1999).

R. Hartshorne, Algebraic Geometry, Springer 1977.

K. Karu, Local strong factorization of birational maps, J. Alg. Geom., 14 (2005), pp. $165-175$. 
R. Morelli, The birational geometry of toric varieties, J. Alg. Geometry, 5 (1996), pp. $751-782$.

J. Włodarczyk, Toroidal varieties and the weak factorization theorem, Invent. Math., 154 (2003), pp. 223-331.

Problem 37**. Normalization. This is not really a well-posed mathematical exercise, but merely an attempt we found inspiring.

Find a refinement of the notion of integral closure of rings and normal varieties which ensures smoothness in codimension 2.

Note. Let $A$ be a finitely generated $K$-algebra which is a domain, and let $A^{\prime}$ be its integral closure in the quotient field of $A$. If $A$ is the coordinate ring of a variety $X, A^{\prime}$ describes the normalization $X^{\prime}$ of $X$. It is well known (see e.g. Mumford) that the singular locus of $X^{\prime}$ has codimension $\geq 2$ in $X$.

The refinement of the integral closure could not be just another overring of $A^{\prime}$, because the normalization is already maximal among all birational affine varieties with a proper birational morphism down to the normalized variety.

A positive answer to the problem would yield a one step resolution of surfaces, as the normalization does for curves. It is conceivable that such a concept had again to do with extension properties as is the case for weakly holomorphic functions on normal varieties. Note here that the extension or integration of differential forms in the context of the singular Frobenius Theorem often requires that the exceptional set has codimension $\geq 3$.

References. T. De Jong, G. Pfister, Local Analytic Geometry, Advanced Lectures Math., Vieweg 2000.

B. Malgrange, Frobenius avec singularités. I, Codimension un. Publ. Math. Inst. Hautes Études Sci., 46 (1976), pp. 163-173.

R. Moussu, Sur l'existence d'intégrales premières, Ann. Inst. Fourier, 26 (1976), pp. $171-220$.

D. Mumford, The Red Book of Varieties and Schemes, Lecture Notes Math., 1358, Springer 1999.

O. Zariski, P. Samuel, Commutative Algebra, Graduate Texts Math., 28, Springer 1975 .

Problem 38. Multiplicity and localization. This is an exercise in commutative algebra.

Let $(R, m)$ be a regular local ring and $I$ a non zero ideal in $R$. Let $\widehat{R}$ denote the completion of $R$ and set $\widehat{I}=I \cdot \widehat{R}$. For a prime ideal $p$ in $R$, denote by $S$ the localization of $R$ at $p$, and by $J$ the ideal generated by $I$ in $S$. Define the order ord $(I)$ of $I$ as the maximal power of $m$ containing $I$, and similarly for $J$.

(a) Show that $\operatorname{ord}(\widehat{I})=\operatorname{ord}(I)$.

(b)* Show that ord $(J) \leq \operatorname{ord}(I)$.

Note. The inequality of (b) is due to Zariski. To prove it, reduce first to the case where $R / p$ has dimension 1 and then use (a) and resolution of curves. You may also consult Hironaka, Thm. III. 3.1.

REFEREnCEs. H. Hironaka, Resolution of singularities of an algebraic variety over a field of characteristic zero, Ann. Math., 79 (1964), pp. 109-326.

Problem 39*. Adjoints. Adjoint functions are a classical computational tool in birational geometry. The arithmetic and geometric genus of $X$ can be read off from 
the Hilbert function of the ideal of adjoint functions. Similarily, the plurigeni of $X$ are related to higher adjoint ideals. The homogenuous parts of the adjoint ideals can be used to define canonical maps, minimal models, and Mori fibrations. For singular hypersurfaces, the computation of adjoint function requires some sort of resolution. Invent a method for computing adjoints without resolution of singularities.

Note. For curves, computing of adjoints is basically equivalent to computing the normalization. For surfaces, there is an efficient algorithm computing adjoints, available in the computer algebra system Magma. It requires the computation of "pieces of a resolution", which are analytically isomorphic to some resolution of the singularities of the given surfaces.

References. T. Beck, J. Schicho, Adjoint computation for hypersurfaces using formal desingularizations, J. Algebra, 320 (2008), pp. 3984-3996.

P. Blass, J. Lipman, Remarks on adjoints and arithmetic genera of algebraic varieties, Amer. J. Math., 101 (1979), pp. 331-336.

Problem 40. Topology of plane curve singularities. The following questions are motivated by "numerical analysis" of the singularities of a plane algebraic curve. What can be said about the singularities if the coefficients of the defining polynomial is known only up to a small numerical error?

Intersecting a complex curve singularity $(X, 0) \subset\left(\mathbb{C}^{2}, 0\right)$ with a sufficiently small $\varepsilon$-sphere around $p$, one obtains a link that classifies the topological type of the singularity. The links that can be obtained in this way are called algebraic links.

(a)* Can the link become non-algebraic when the radius of the sphere becomes larger?

(b)* Assume that $(X, 0)$ intersects a sphere with radius $\varepsilon>0$ in an algebraic link $L$. Can we continously deform $(X, 0)$ to a curve singularity with link $L$, such that the induced deformation of the intersection with the sphere is an isotopy of links?

References. M. Borodzik, Deformations of singularities of plane curves, Topological approach, preprint, arXiv 0907.4129.

M. Borodzik, Morse theory for plane algebraic curves, preprint, arXiv 1101.1870.

M. Hodorog, M. Mourrain, B., J. Schicho, A symbolic-numeric algorithm for computing the Alexander polynomial of a plane curve singularity, Proc. SYNASC 2010, IEEE Trans. 2010.

Note added in proof. Bernard Teissier has kindly informed us that question (b) of Problem 34 has a negative answer which is due to H. Whitney. The germ of the hypersurface defined by the equation

$$
(y-x)(y-2 x)(y-3 x)(y-(t-1) x)\left(y-\left(e^{t}-1\right) x\right)=0
$$

cannot be isomorphic to an algebraic variety, since among the five cross ratios of four of the lines respectively, two at least are algebraically independent as functions of the parameter $t$.

In the real analytic setting and taking homeomorphisms, the problem is due to René Thom. In the Lipschitz case, similar questions have been investigated by Valette.

Teissier has conjectured the following: Any reduced complex analytic germ is isomorphic to the intersection - inside a suitable non singular space - of an algebraic germ of higher dimension with a smooth analytic germ, the second being transversal to the canonical Whitney stratification of the first. 
